\title{
Compensatory Plasticity at an Identified Synapse Tunes a Visuomotor Pathway
}

\author{
Stephen M. Rogers, ${ }^{1}$ Holger G. Krapp ${ }^{1,2}$ Malcolm Burrows, ${ }^{1}$ and Thomas Matheson ${ }^{1,3}$ \\ ${ }^{1}$ Department of Zoology, University of Cambridge, Cambridge CB2 3EJ, United Kingdom, ${ }^{2}$ Department of Bioengineering, Imperial College London, \\ London SW7 2AZ, United Kingdom, and '3Department of Biology, University of Leicester, Leicester LE1 7RH, United Kingdom
}

\begin{abstract}
We characterized homeostatic plasticity at an identified sensory-motor synapse in an insect, which maintains constant levels of motor drive as locusts transform from their solitarious phase to their gregarious swarming phase. The same mechanism produces behaviorally relevant changes in response timing that can be understood in the context of an animal's altered behavioral state. For individual animals of either phase, different looming objects elicited different spiking responses in a visual looming detector interneuron, descending contralateral movement detector (DCMD), yet its synaptic drive to a leg motoneuron, fast extensor tibiae (FETi), always had the same maximum amplitude. Gregarious locust DCMDs produced more action potentials and had higher firing frequencies, but individual postsynaptic potentials (PSPs) elicited in FETi were half the amplitude of those in solitarious locusts. A model suggested that this alone could not explain the similarity in overall amplitude, and we show that facilitation increased the maximum compound PSP amplitude in gregarious animals. There was the same linear relationship between times of peak DCMD firing before collision and the size/velocity of looming objects in both phases. The DCMD-FETi synapse transformed this relationship nonlinearly, such that peak amplitudes of compound PSPs occurred disproportionately earlier for smaller/faster objects. Furthermore, the peak PSP amplitude occurred earlier in gregarious than in solitarious locusts, indicating a differential tuning. Homeostatic modulation of the amplitude, together with a nonlinear synaptic transformation of timing, acted together to tune the DCMD-FETi system so that swarming gregarious locusts respond earlier to small moving objects, such as conspecifics, than solitarious locusts.
\end{abstract}

Key words: homeostatic plasticity; sensory-motor integration; DCMD; nonlinear transformation; locust; phenotypic plasticity

\section{Introduction}

Imposed disturbances of neuronal activity can lead to compensatory changes in synapses, neurons, or networks through processes collectively known as homeostatic plasticity (Turrigiano, 1999; Turrigiano and Nelson, 2000, 2004). We characterize homeostatic plasticity at an identified synapse in two morphs of the same species. The morphs differ physiologically, morphologically, and behaviorally, adapting them to changing environmental conditions and population density. A sensory-motor transfer function at the synapse, thought to be important in collision avoidance behavior, is homeostatically modulated so that the maximum response amplitude is maintained. Nevertheless, response timing is modified by this plasticity in a way that can be directly related to behavioral adaptations appropriate for their different environments.

The desert locust Schistocerca gregaria shows a radical, but fully reversible, form of phenotypic plasticity (phase change) that involves changes in behavior, morphology physiology, neurochemistry and neuronal function (Uvarov, 1966, 1977; Simpson

Received 0ct. 24, 2006; revised March 20, 2007; accepted March 21, 2007.

This work was supported by the Biotechnology and Biological Sciences Research Council (United Kingdom). We thank Jeremy Niven for insightful comments on this manuscript.

Correspondence should be addressed to Stephen M. Rogers, Department of Zoology, University of Cambridge, Downing Street, Cambridge CB2 3EJ, UK. E-mail: smr34@cam.ac.uk.

DOI:10.1523/JNEUROSCI.4615-06.2007

Copyright $\odot 2007$ Society for Neuroscience $\quad$ 0270-6474/07/274621-13\$15.00/0 et al., 1999; Matheson et al., 2004; Rogers et al., 2004). Locusts generally occur at low population densities $\left(<3 / 100 \mathrm{~m}^{2}\right)$ in the "solitarious phase." They are cryptic in appearance and behavior, they are sedentary, and they actively avoid other locusts. In contrast, locusts in the notorious swarming "gregarious phase" occur at high population densities $\left(>100,000 / 100 \mathrm{~m}^{2}\right)$ and are conspicuous, very active, and attracted toward other locusts, forming cohesive groups. Phase change in locusts provides a powerful model system with which to analyze a whole suite of reversible behavioral and neuronal plasticities.

The responses of an identified visual interneuron, the descending contralateral movement detector (DCMD), differ markedly between the solitarious and gregarious phases (Matheson et al., 2004). Spikes in DCMD follow spikes in its presynaptic neuron, the lobula giant movement detector (LGMD) (O'Shea and Williams, 1974) with a 1:1 relationship. LGMD produces the highest spiking frequencies to looming visual objects on collision course with the locust, which elicit spike bursts of increasing frequency as the approaching object expands across the visual field (Schlotterer, 1977; Rind and Simmons, 1992; Simmons and Rind, 1992). DCMD conveys the output of LGMD from the brain to interneurons and motoneurons involved in flight steering and jumping (Burrows and Rowell, 1973; Pearson et al., 1980; Simmons, 1980; Santer et al., 2005a, 2006).

We analyzed the response of one motoneuron that receives monosynaptic input from DCMD, the metathoracic fast extensor 
tibiae (FETi) (Burrows and Rowell, 1973; Pearson and Goodman, 1979) and compared the tuning of this synapse between phases. We show that differences in the firing frequency of DCMD between phases are accompanied by considerable differences in the properties of the DCMD-FETi synapse. These phase-related differences in DCMD firing rate, postsynaptic potential (PSP) amplitude, and synaptic facilitation serve to counteract each other so that the compound PSPs in FETi have the same amplitude in both phases. The altered properties of the DCMD-FETi synapse, however, advance the timing of the peak amplitude of the compound PSP relative to collision for small/fast objects in gregarious locusts compared with solitarious locusts.

\section{Materials and Methods}

Animal rearing. Experiments were performed on desert locusts (Schistocerca gregaria Forskål) of both sexes 1-2 weeks after they had moulted to adulthood. Gregarious phase locusts were obtained from cultures at the Universities of Cambridge and Oxford, UK, which are maintained under high population densities $\left(\sim 4000 / \mathrm{m}^{3}\right)$, and fed on seedling wheat and wheat bran flakes. Phase change occurs over several different time scales from hours to generations, with phase state transmitted epigenetically to offspring. Solitarious animals regain gregarious behavioral characteristics within as little as $4 \mathrm{~h}$ of recrowding, but other morphological and physiological reversions back to the gregarious state take longer, accumulating slowly over the lifespan or even over the course of several generations of isolation or crowding. The properties of DCMD (Matheson et al., 2004) appear to change within one generation of isolation of gregarious locusts (S. M. Rogers, personal observation). The solitarious phase animals used were the third-generation descendents of locusts from the Oxford gregarious colony that had each been reared in individual cages under visual, olfactory, and tactile isolation from other locusts, using husbandry procedures described by Roessingh et al. (1993). Such animals exhibit strongly solitarious morphological, physiological, and behavioral phenotypes (Simpson et al., 1999). Cambridge animals were maintained at $37-25^{\circ} \mathrm{C}$ under an 18/6 h light/dark photoperiod, whereas Oxford animals experienced a $12 / 12 \mathrm{~h}$ photoperiod at a constant temperature of $30 \pm 2^{\circ} \mathrm{C}$.

Dissection and recording. Animals were mounted ventral side up in a plastic holder and restrained using Plasticine such that the right eye had an unimpeded visual field. The head was fixed into position with bees wax so that the long axis of the compound eye was vertical and the cervical membrane stretched. The cervical cuticle and underlying air sacs were removed to expose the ventral nerve cord. A pair of bipolar 50 - $\mu \mathrm{m}$-diameter silver hook electrodes were inserted under the left cervical connective and
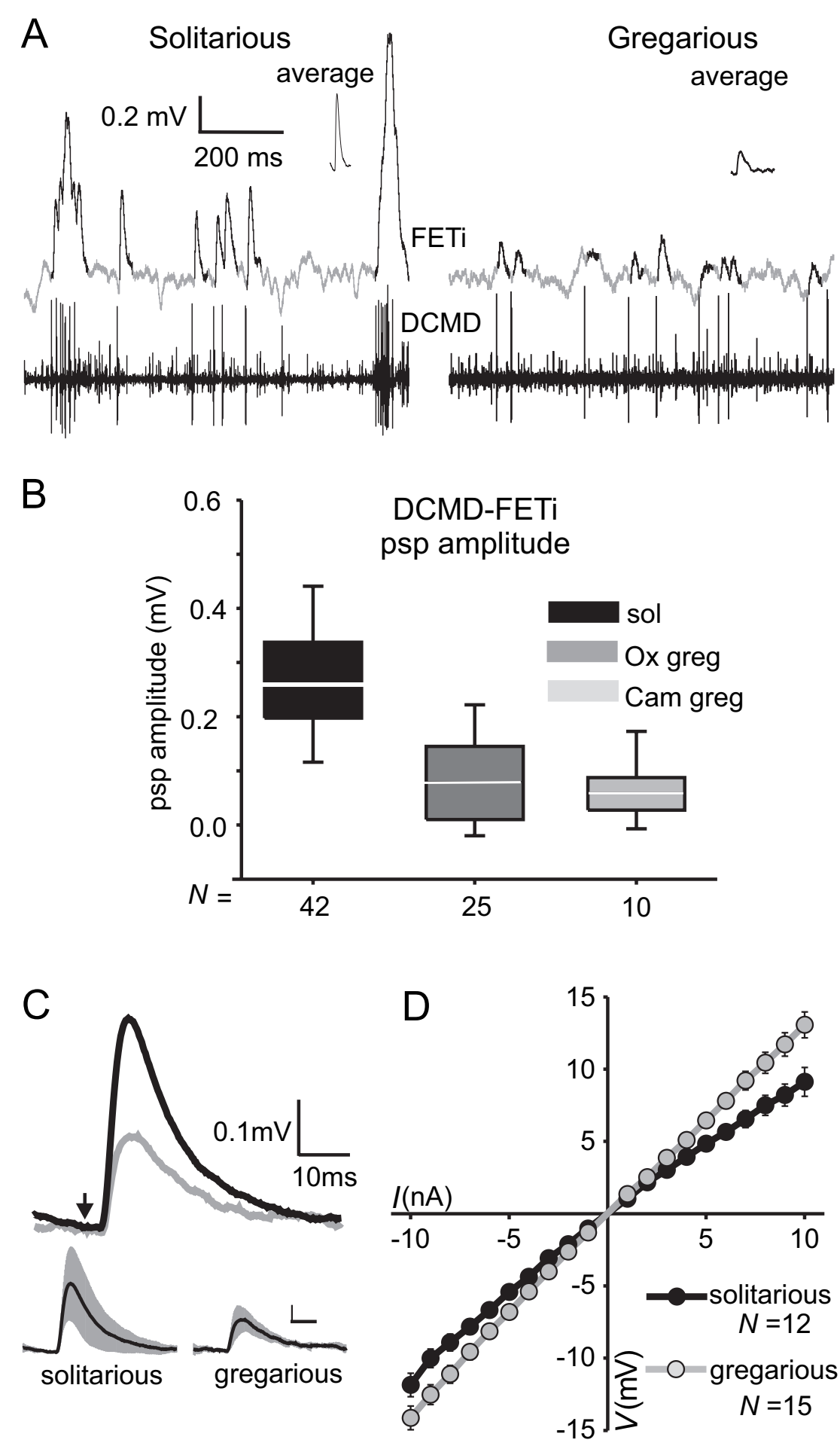

Figure 1. PSPs evoked by DCMD in FETi differ between locust phases. $A$, Recordings of DCMD and FETi in solitarious (left) and gregarious (right) locusts during stimulation by an object moving slowly across the visual field. DCMD-evoked PSPs in FETi are shown in black to distinguish them from background synaptic inputs. Averages of 50 PSPs in these recordings are shown as insets. B, Box plots showing medians (white line), 50\% data range (boxes), and 90\% data range (whiskers) for DCMD-evoked FETi PSP amplitudes for solitarious (sol) and two genetically distinct groups of gregarious locusts ( $0 x$ greg, $0 x f o r d$ gregarious; Cam greg, Cambridge gregarious). C, Average evoked PSPs in FETi from nine different solitarious (black) and nine different gregarious (gray) locusts. The small traces below show the SD (between locusts), with the scale bar at the same values as above. The arrow indicates the time of the triggering DCMD spike. D, Voltage response of FETi to current injections in the soma of gregarious (gray) and solitarious (black) locusts. Data are mean \pm SEM. 
were insulated using a 10:1 petroleum jelly:liquid paraffin mixture. DCMD spikes were recorded using an AC amplifier (1000× amplification; bandwidth, 50-10,000 Hz). DCMD has the largest amplitude action potentials in extracellular recordings of a cervical connective so spike times could be obtained simply by using an amplitude threshold. The response of DCMD to visual stimuli delivered to the contralateral eye is unique in these recordings, so there was no ambiguity in identifying the neuron. A second window in the cuticle was made in the ventral surface of the thorax to expose the mesothoracic and metathoracic ganglia. A wax-coated silver platform, which acted as the indifferent electrode, was inserted under these ganglia. Great care was taken not to damage any of the tracheae supplying the metathoracic ganglion as any interruption of the air supply rapidly abolished the activity of the DCMD-FETi synapse. Intracellular recordings were made from the soma of FETi using thin-walled glass microelectrodes filled with $1 \mathrm{M}$ potassium acetate (resistance of 20-30 M $\Omega$ ). FETi was identified by its location and the occurrence of an antidromic spike in the soma evoked by electrical stimulation of the extensor tibiae muscle of the hindleg. Recordings of the input resistance of FETi were made using single electrode clamp with either an Axoclamp-2a amplifier (Molecular Devices, Union City, CA) or an NPI Electronics (Tamm, Germany) amplifier in discontinuous current clamp mode set with switching rates between 5 and $10 \mathrm{kHz}$.

Data were recorded on a computer using a Cambridge Electronic Design (Cambridge, UK) micro1401 interface and Spike 2 (version 5.08; Cambridge Electronic Design) software. DCMD signals were sampled at $25 \mathrm{kHz}$ and FETi at $6.16 \mathrm{kHz}$. Stable recordings were maintained from 40 min to several hours. Data from recordings in which the height of the antidromic spike in FETi had decreased by $>20 \%$ over the course of an experiment were not analyzed. Experiments were performed at $24-27^{\circ} \mathrm{C}$.

Visual stimulation. In an initial set of experiments to determine the relative sizes of single PSPs in solitarious and gregarious locusts, a small probe (a $5 \mathrm{~mm}$ square flag on a stick) was moved by hand in front of the visual field at a distance of $20-200 \mathrm{~mm}$ from the eye. This stimulated DCMD to fire spikes at low frequency $(1-5 \mathrm{~Hz})$ and elicited corresponding PSPs in FETi ( $n=42$ solitarious locusts, 25 locusts from the Oxford gregarious colony, and 10 from a genetically unrelated gregarious colony maintained at the Department of Zoology, Cambridge University, Cambridge, UK).

The principal experiments analyzed the response of DCMD-FETi to looming stimuli and used the controlled visual stimulation protocol developed by Gabbiani et al. (1999) as modified by Matheson et al. (2004). Locusts were placed $60 \mathrm{~mm}$ from the $100 \times 120 \mathrm{~mm}$ screen of a Tektronix (Wilsonville, OR) CRT (model 608) with the right eye aligned precisely to its center in both vertical and horizontal directions. The monitor had a refresh rate of $185 \mathrm{~Hz}$ and a spatial resolution of $0.5 \mathrm{~mm}$ (angular resolution at $60 \mathrm{~mm}, 0.48^{\circ}$ ) well above the temporal resolution and below the spatial resolution of the locust compound eyes (Wilson, 1975; Horridge, 1978; Howard, 1981). The monitor was controlled by a Picasso image synthesizer (Innisfree, Cambridge, MA) driven by the output of the Cambridge Electronic Design data acquisition system.

The stimuli simulated the last $5 \mathrm{~s}$ of approach before collision of a dark square object against a light background (52\% contrast). The objects appeared in the center of the screen in line with the eye and expanded to subtend an angle of $80^{\circ}$ to the eye. On reaching maximum size just before collision, the stimulus remained static for $500 \mathrm{~ms}$ and then disappeared. The rate of expansion of an approaching object depends on both its size and velocity but can be defined by a single ratio, $l /|v|$, where $l$ is the half size of the object and $v$ is the approach velocity of the object (Gabbiani et al., 1999). We used stimuli of $l /|v|=2.5,5,10,20$, and $40 \mathrm{~ms}$, which corresponds to, for example, square objects 10-160 mm across, approaching at a constant velocity of $2 \mathrm{~m} \mathrm{~s}^{-1}$, or alternatively to a $50 \mathrm{~mm}$ square approaching at $10-0.05 \mathrm{~m} \mathrm{~s}^{-1}$. Because of their larger size (or lower approach velocity), objects with a larger $l /|v|$ first appear on the screen at a larger size (ranging from $0.9^{\circ}$ visual angle for $l /|v|=2.5 \mathrm{~ms}$ to $1.4^{\circ}$ for $l /|v|=40 \mathrm{~ms}$ ) and expand more slowly during the simulated approach (Gabbiani et al., 1999).

After establishing an intracellular recording of FETi, all locusts were left for 8-10 min to allow DCMD to fully dishabituate before the start of the experiment (our personal observation). The analyses of responses to

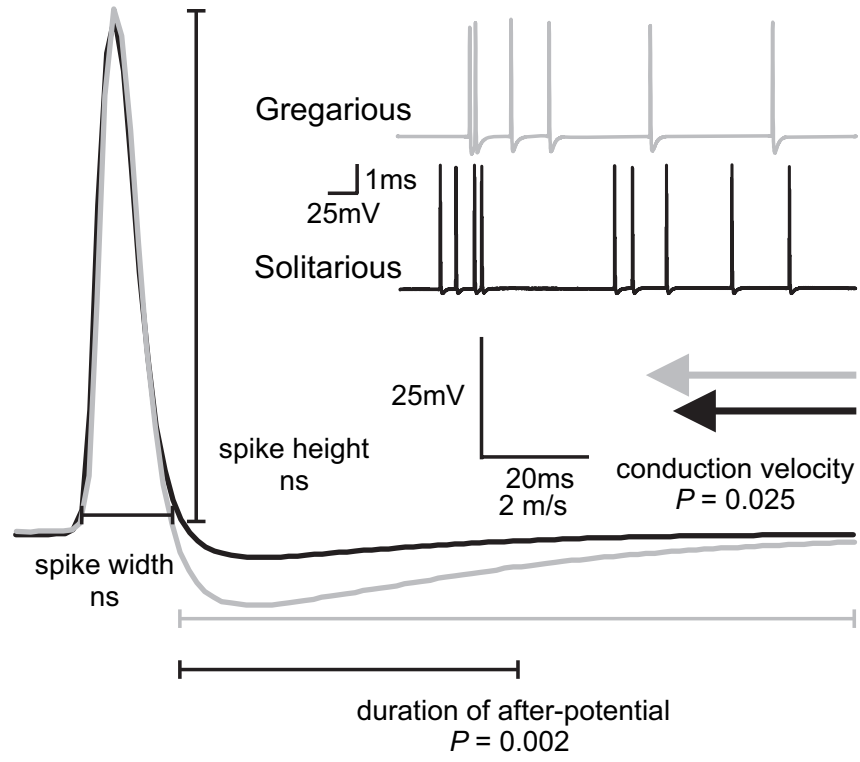

Figure 2. Characteristics of DCMD measured using intracellular recording. $A$, Main figure, examples of a mean (30 sweeps) DCMD spike from a gregarious (gray) and solitarious (black) locust obtained from low firing rate $(<10 \mathrm{~Hz})$ conditions. The inset shows a section of the original recordings. Mean DCMD action potential width, height, resting potential, duration of hyperpolarizing afterpotential, and conduction velocity (gray and black arrows) were compared between phases.

looming stimuli are based on data gathered from nine gregarious and nine solitarious phase locusts. All of these were stimulated by $l /|v|=20$ $\mathrm{ms}$ for 10 approaches at $1 \mathrm{~min}$ intervals to analyze the effect of DCMD habituation on FETi response. After this, all locusts except for one solitarious individual were tested with single stimuli of $l /|v|=5-40 \mathrm{~ms}$ presented in random order at $8 \mathrm{~min}$ intervals. In a few experiments, $l /|v|$ $=2.5 \mathrm{~ms}$ was also used. To examine the amplitudes of individually evoked PSPs in FETi, DCMD was also stimulated with the same small visual stimulus used in the preliminary experiments described above.

Data analysis. Times of DCMD spikes were expressed as times relative to collision (which occurred at time $=0$ ). The time course of the DCMD response was analyzed by convolving a Gaussian smoothing filter with the DCMD spike times using Spike 2 software. The Gaussian distribution had a SD of $20 \mathrm{~ms}$ and amplitude of $25.1 \mathrm{~Hz}$. The use of Gaussian smoothing avoids artifacts caused by the temporal binning usually used to generate peristimulus time histograms (Gabbiani et al., 1999). It also removes problems associated with doublet spikes in DCMD producing transiently high instantaneous frequencies and emphasizes the time at which DCMD rises to a sustained high firing rate (see Fig. 3). Peak DCMD spike frequencies and the time of peak firing relative to collision were obtained from these smoothed data. Linear regressions were fitted to the data to compare the effects of different values of $l /|v|$ on the responses of DCMD and FETi for each individual animal. The coefficients of these regressions were then compared using $t$ tests (SPSS version 10.0; SPSS, Chicago, IL) to determine the significance of any differences between phases, thus avoiding the problem of repeated measurements from individual locusts.

Model. We modeled the expected response of FETi to stimulation by DCMD by convolving a single PSP template with the times of DCMD spikes. The PSP templates were generated for both solitarious and gregarious locusts by fitting a sigmoid rising function and simple exponential decay function to an averaged PSP calculated from data from all successful recordings. Average PSPs were generated from $n=40-50$ averaged single PSPs obtained from low-frequency stimulation of DCMD from $n=9$ locusts of each phase. Latencies from a DCMD spike to the start of the rising phase of the PSP template were adjusted to match the experimentally measured latencies for each recording. Unless otherwise stated, all values quoted are means $\pm 1 \mathrm{SD}$.

Testing for synaptic plasticity at the DCMD-FETi synapse. DCMD was 

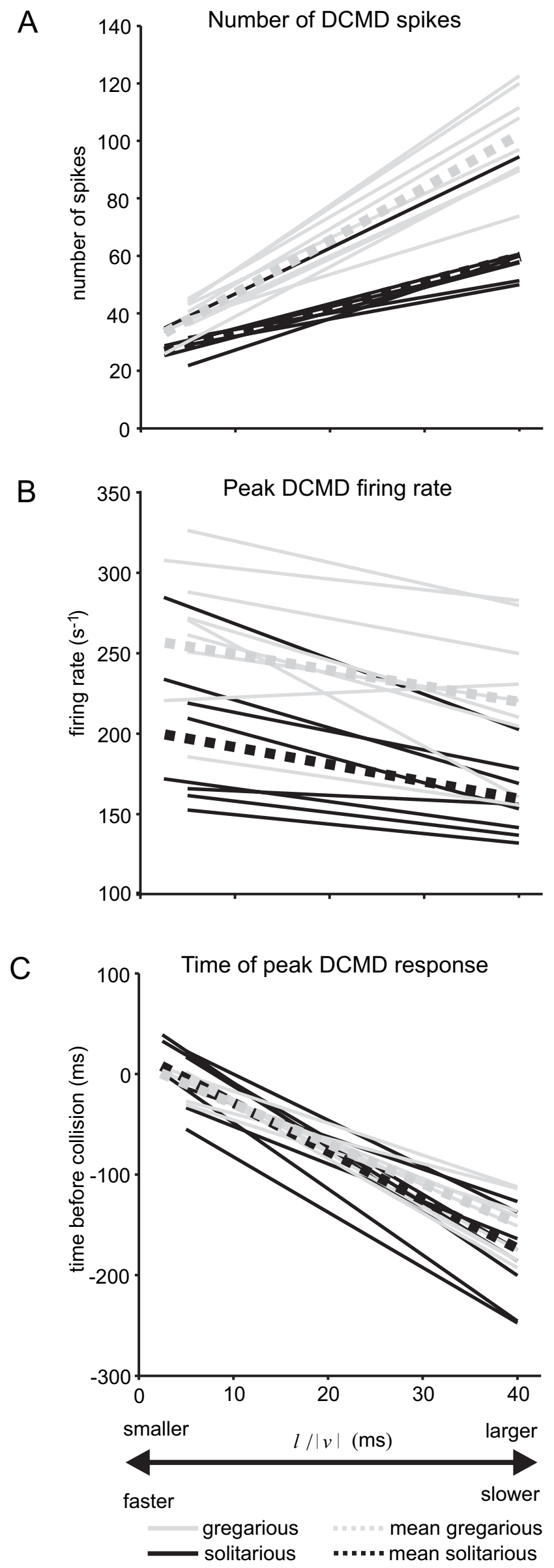

electrically stimulated to test for facilitation at the DCMD-FETi synapse. We added the following: the large size of the DCMD axon meant that it was difficult to inject enough current through an intracellular electrode to reliably elicit spiking. Therefore, we used extracellular stimulation applied to the cervical connective to drive DCMD. A pair of stimulating hook electrodes was placed around the cervical connectives positioned such that there was a good contact with the dorsomedial surface of the connective, near the axon of DCMD. A second pair of extracellular electrodes was placed around the connectives connecting the prothoracic and mesothoracic ganglia to record the spike evoked by the electrical stimulus. FETi was recorded intracellularly as detailed above. The stimulus voltage was adjusted so that it just evoked a spike as recorded by the extracellular electrodes downstream. In most preparations, this spike shape closely resembled that of DCMD, which, because of its large size and proximity to the stimulation site, was preferentially stimulated at the threshold voltage (2-3 V, depending on the preparation). Because of the risk of stimulating other axons adjacent to DCMD, which could, in principle, also synapse onto FETi, we compared the PSPs elicited by electrically driven spikes with PSPs elicited by visually stimulating DCMD to ensure that they had the same amplitude and latency (see Results). At threshold stimulation voltage, this was invariably the case, and it was therefore highly likely that both visually and electrically elicited PSPs in FETi derived from DCMD. Because DCMD has the largest axon diameter in the connective and therefore presumably the fastest conduction velocity, PSPs driven by any other axons would have had a longer latency, which was never the case. Preparations in which the evoked extracellular spike was dissimilar to DCMD and did not elicit PSPs in FETi were not analyzed. Five locusts of each phase were tested with stimulus trains consisting of 10 pulses each $0.05 \mathrm{~ms}$ in duration spaced at $30 \mathrm{~ms}$ intervals. The stimulus train was repeated $20-100$ times at $50 \mathrm{~s}$ intervals.

\section{Results}

DCMD-evoked PSPs in FETi are larger in solitarious locusts DCMD spikes evoked PSPs in FETi (Fig. 1), with a consistent latency for individual animals $(4.2 \pm 0.85 \mathrm{~ms}$ in solitarious and $3.8 \pm 0.43 \mathrm{~ms}$ in gregarious locusts not significantly different between phases; $t_{11.8}=-1.08 ; p=0.301 ; n=18$ locusts). The long latency for this monosynaptic connection is attributable to the long conduction time down the DCMD axon from the recording site in the neck. DCMD responded at low frequencies to a small object moving randomly across the visual field and when firing at $<35$ spikes $\mathrm{s}^{-1}$, the resulting PSPs in FETi did not summate (Fig. $1 A$ ). In gregarious locusts, these single PSPs were small and often difficult to discern amid the ongoing synaptic inputs from other sources, but they could be retrieved by DCMD spiketriggered averages (Fig. $1 A$, inset top). In contrast, in solitarious animals, the single PSPs in FETi were much larger and always apparent above background synaptic inputs.

In an initial experiment, solitarious phase locusts from the Oxford colony were compared with two groups of gregarious locusts: one from the parent colony of the solitarious locusts at Oxford and the other a genetically distinct population maintained at Cambridge (Fig. $1 B$ ). In solitarious locusts, the average PSP amplitude $(0.37 \pm 0.13 \mathrm{mV})$ was over twice the amplitude of the PSPs evoked in either of the gregarious populations, which were similar to each other (Oxford gregarious, $0.18 \pm 0.09 \mathrm{mV}$; Cambridge gregarious, $0.17 \pm 0.05 \mathrm{mV}$; $\operatorname{ANOVA}, F_{(2,74)}=27.5$; $p<0.001)$. Similar values for single PSP amplitudes in FETi were obtained in all of the experiments where the responses of DCMD-

\section{$\leftarrow$}

Figure 3. Response characteristics of $D C M D$ to looming stimuli differ with changing $/ /|v|$ and between phases. $\boldsymbol{A}$, Number of spikes. $\boldsymbol{B}$, Peak-firing rate. $\boldsymbol{C}$, The time of peak DCMD response relative to collision. The graphs show regressions fitted to data obtained from individual locusts (black, solitarious; gray, gregarious). The dotted lines show the mean regressions for each phase. 

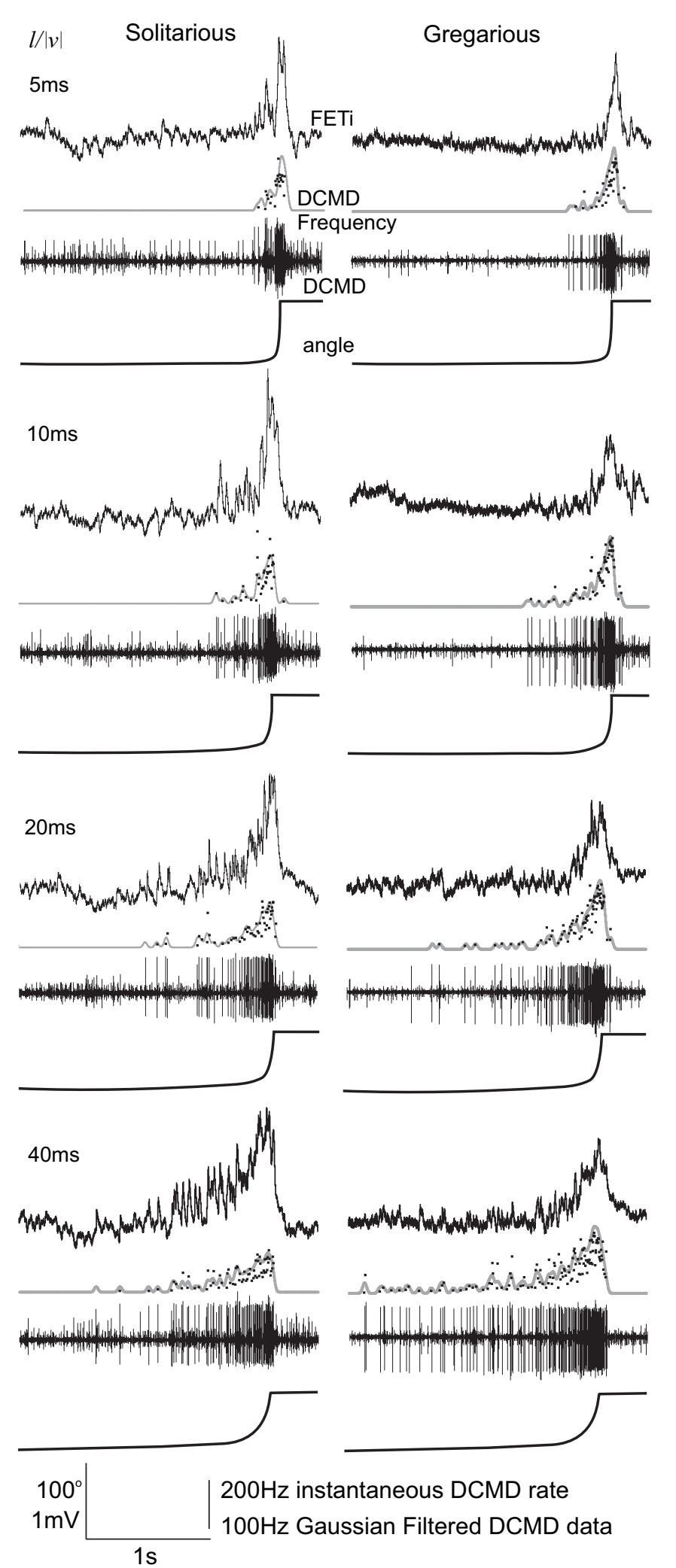

Figure 4. Looming stimuli evoke bursts of spikes in DCMD and a compound PSP in FETi. The left column shows a representative recording from a solitarious locust during stimulation with looming objects of different values of $I /|v|$, whereas the right column shows corresponding responses in a gregarious locust. The data are from the top: the intracellular FETi response, the instantaneous DCMD firing rate (dots) and the Gaussian smoothed data (gray lines), the extracellular response of DCMD; and bottom, the angle subtended by the stimulus at the retina.
FETi to looming objects were tested (Fig. 1C) (solitarious PSP amplitude, $0.41 \pm 0.18 \mathrm{mV}$; gregarious PSP amplitude, $0.19 \pm$ $\left.0.08 \mathrm{mV} ; t_{16}=3.6 ; p=0.003\right)$. The time constant of PSP decay did not differ significantly between phases (solitarious, $12.7 \pm 6.0$ ms; gregarious, $\left.17.3 \pm 8.3 \mathrm{~ms} ; t_{16}=-1.348 ; p=0.196\right)$.

We therefore sought possible mechanisms that could explain the differences in PSP amplitudes between phases. First, we measured the input resistance of FETi, which was significantly lower in solitarious locusts than in gregarious as measured in the soma (Fig. $1 D$ ) (to positive current 9.3 $\pm 0.3 \mathrm{M} \Omega$ in solitarious, $13.3 \pm$ $0.39 \mathrm{M} \Omega$ in gregarious; $t_{22}=2.73 ; p=0.012$ ). From this result, it would be expected that DCMD-evoked PSPs recorded in the soma of FETi would be smaller in solitarious than gregarious locusts, all other factors being equal. Antidromic spikes in FETi, which are passively reflected in the soma, for example, were slightly but not significantly smaller in solitarious locusts (solitarious, $9.3 \pm 2.09 \mathrm{mV}$; gregarious, $9.9 \pm 3.14 \mathrm{mV} ; t_{16}=0.512$; $p=0.616)$. The observed DCMD-PSP amplitude data in FETi are the opposite of this, however. Second, the time constant of FETi was also similar between phases (average across all experiments, $11.5 \pm 2.4 \mathrm{~ms}$ ). A third possible mechanism would be differences in the waveform of DCMD spikes, because change in presynaptic spike amplitude or width can alter the strength of synaptic transmission (Jackson et al., 1991; Burrows and Matheson, 1994; Nusbaum et al., 1997; Niven and Burrows, 2003). Intracellular recordings of DCMD made in its axon as it leaves the mesothoracic ganglion (Fig. $2 \mathrm{~A}$, waveform) revealed that spike width (solitarious, $1.2 \pm 0.32 \mathrm{~ms}$; gregarious, $1.4 \pm 0.49 \mathrm{~ms} ; t_{20}=0.746 ; p=$ 0.465 ), spike height (solitarious, $61.7 \pm 25.1 \mathrm{mV}$; gregarious, $54.8 \pm 29.6 \mathrm{mV} ; t_{20}=0.874 ; p=0.573$ ), and resting potential (solitarious, $-65.2 \pm 7.4 \mathrm{mV}$; gregarious, $-63.0 \pm 13.6 \mathrm{mV} ; t_{16}$ $=0.41 ; p=0.69)$ were similar in both phases. In gregarious locusts, however, the duration of the spike afterpotential was significantly longer $\left(4.4 \pm 1.4\right.$ compared with $2.9 \pm 0.4 \mathrm{~ms} ; t_{14.4}$ $=3.69 ; p=0.002$ ). Conduction velocity was also $12 \%$ faster in gregarious locusts (measured between the cervical extracellular recording and intracellular recordings in the mesothoracic ganglion; gregarious $3.9 \pm 0.48 \mathrm{~m} / \mathrm{s}$, solitarious $3.4 \pm 0.36 \mathrm{~m} / \mathrm{s} ; t_{20}=$ $2.4 ; p=0.025$ ) (Fig. 2). Therefore, we conclude that the larger DCMD-FETi PSP amplitude in solitarious locusts arises from alterations in the transmission strength of the synapse itself, not from alterations in the passive membrane properties of either neuron.

\section{Responses of DCMD to looming stimuli}

DCMD responds to looming stimuli with a burst of spikes that escalates in frequency as the retinal size of the approaching object increases in an expansive nonlinear way. The spike frequency reaches a maximum and then declines, generally before the end of object expansion, depending on the size $(l)$ and/or velocity $(v)$ of the object. The response properties of DCMD differed between phases and with $l /|v|$. Increasing $l /|v|$ (i.e., increasing object size and/or decreasing approach velocity) evoked more DCMD spikes in both phases (Fig. $3 A$ ). In gregarious locusts, however, spike numbers were higher for all values of $l /|v|$ than in solitarious, with the difference between phases growing as $l /|v|$ increased (Fig. 3A, Table 1). For example, at $l /|v|=5 \mathrm{~ms}, 37 \pm 4.7 \mathrm{DCMD}$ spikes were elicited in gregarious locusts compared with $28 \pm 6.4$ spikes in solitarious (a difference of 32\%), but for objects of $l /|v|$ $=40 \mathrm{~ms}$, the difference had increased to $70 \%$ (99 \pm 16.7 DCMD spikes in gregarious compared with $59 \pm 14.6$ spikes in solitarious). Conversely, increasing $l /|v|$ led to a decrease in the peak firing rate of DCMD (Fig. $3 B$ ). Gregarious locusts had consis- 
Table 1. Results of $t$ tests analyzing the differences between solitarious and gregarious locusts of coefficients obtained from linear regressions of characteristics of DCMD and FETi against $I /|v|$

\begin{tabular}{|c|c|c|c|c|c|c|}
\hline & & Value solitary & Value gregarious & $t$ & Degrees of freedom & $p$ \\
\hline \multirow[t]{2}{*}{ Number of DCMD spikes (number) } & Intercept & $25.4 \pm 4.3$ & $31.3 \pm 4.4$ & -2.824 & 15 & 0.013 \\
\hline & Gradient & $0.9 \pm 0.3$ & $1.7 \pm 0.4$ & -4.817 & 15 & $<0.001$ \\
\hline \multirow[t]{2}{*}{ Peak DCMD firing rate (spikes $s^{-1}$ ) } & Intercept & $204 \pm 46.7$ & $271 \pm 44.1$ & -3.028 & 15 & 0.008 \\
\hline & Gradient & $-1.1 \pm 0.66$ & $-1.2 \pm 0.9$ & 0.243 & 15 & 0.811 \\
\hline \multirow[t]{2}{*}{ Time DCMD maximum (ms before collision) } & Intercept & $20.8 \pm 31.8$ & $11.3 \pm 16.2$ & -0.764 & 10.1 & 0.463 \\
\hline & Gradient & $-3.9 \pm 1.2$ & $-2.9 \pm 1.1$ & 1.784 & 15 & 0.095 \\
\hline \multirow[t]{2}{*}{ Compound PSP amplitude (mV) } & Intercept & $1.8 \pm 0.4$ & $1.5 \pm 0.8$ & 0.864 & 15 & 0.401 \\
\hline & Gradient & $-0.002 \pm 0.009$ & $0.007 \pm 0.03$ & -0.918 & 15 & 0.373 \\
\hline
\end{tabular}

tently higher peak firing rates at all values of $l /|v|$, but the relationship between firing rate and $l /|v|$ was similar for both phases (Fig. $3 B$, Table 1). For example, at $l /|v|=5 \mathrm{~ms}$, the mean peak DCMD firing rate was $266 \pm 44$ spikes s $^{-1}$ for gregarious locusts compared with $200 \pm 45$ spikes s $^{-1}$ for solitarious locusts. By $l /|v|=$ $40 \mathrm{~ms}$, the peak rate had fallen to $221 \pm 44$ spikes s $^{-1}$ and $158 \pm$ 24 spikes $s^{-1}$ respectively, a differential between phases of 33$40 \%$ across the entire stimulus range.

The time of peak DCMD firing relative to collision occurred earlier with increasing $l /|v|$ (Fig. $3 C$ ), and this pattern did not differ between phases. This is in agreement with our previous analyses (Gabbiani et al., 1999; Matheson et al., 2004). The mean time of peak firing relative to collision ranged from $-1.2 \pm 30.2$ $\mathrm{ms}$ for $l /|v|=5 \mathrm{~ms}$ decreasing to $-165 \pm 37 \mathrm{~ms}$ for objects of $l /|v|$ $=40 \mathrm{~ms}$, where negative values are times before collision.

\section{Response characteristics of FETi to looming stimuli}

During the presentation of a looming object, the frequency of DCMD firing increased, and the evoked PSPs in FETi summated to form sharply peaked compound PSPs (Fig. 4; mean values in Fig. 5). The areas underneath the compound PSPs increased with $l /|v|$, corresponding to the increasing number of DCMD spikes (Figs. 4, 5), and the compound PSPs were similar in shape in both phases (Fig. 5, overlaid averages are shown in the right column). The maximum amplitudes of the compound PSPs were also remarkably consistent and did not differ with $l /|v|$ or between phases [mean $1.8 \pm 0.61 \mathrm{mV}$ (Fig. $6 A, E$ ); neither intercepts nor gradients of the regressions were significantly different between phases (Table 1)]. This constancy occurred despite the pronounced differences in DCMD response both between phases and to different visual stimuli, and the considerable phase-related difference in the amplitude of individual DCMD evoked PSPs in FETi (Fig. 1C).

For solitarious locusts, the predicted compound PSP in FETi, made by convolving recorded DCMD spike trains with PSP templates that matched the size and shape of the single PSPs (evoked at a low rate; see Materials and Methods), closely approximated the maximum amplitude and shape of the compound PSPs recorded in FETi (Fig. 6B-E). If the model was a good predictor of the DCMD-FETi synapse, then the ratio of the model single PSP to the maximum convolved PSP should be the same as the ratio between the measured single PSP and the recorded maximum amplitude of the compound PSP. In the case of solitarious locusts, this was indeed the case, as the values cluster around the dotted line indicating ratios of the same value (Fig. $6 C, D$, black symbols). Because both the X and Y data have statistical errors associated with them, the data form a cloud about this line rather than a perfect linear relationship. The gregarious locust data were, for the most part, well to the right of this line of equivalent ratio between model and experimental data, indicating that the measured values for maximum compound PSP amplitude were considerably greater than those predicted from the model and the amplitudes of individual gregarious PSPs (Fig. $6 C, D$, gray symbols). The convolved waveform followed the inflections of the recorded compound PSPs well, but the amplitude of the simulation fell increasingly below the recorded data over the course of a looming stimulus (Fig. $6 \mathrm{~B}$ ).

There were only small differences between the recorded and predicted amplitudes for solitarious locusts (model mean, $1.7 \pm$ $0.60 \mathrm{mV}$; actual mean, $1.8 \pm 0.43 \mathrm{mV}$; model amplitude on average $6 \%$ less than recorded value). In contrast, in gregarious locusts, there were considerable differences between the model value $(1.2 \pm 0.43 \mathrm{mV})$ and actual value $(1.8 \pm 0.74 \mathrm{mV}$; model prediction on average $33 \%$ less than recorded values). A repeated-measures ANOVA comparing the mean expected and recorded amplitudes for each value of $l /|v|$ for each animal indicated that there were significant differences between the predicted and measured maximum amplitudes $\left(F_{(1,64)}=12.7 ; p<\right.$ $0.001)$, as well as a significant interaction with phase $\left(F_{(1,64)}=\right.$ $9.1 ; p=0.004)$, but no interaction with $l /|v|\left(F_{(4,64)}=1.9 ; p\right.$ $=0.119$ ) (i.e., the accuracy of the model did not change according to the size and/or velocity of the stimulus) (Fig. 6, $C$ and $D$, shows the scatter of the data). Analyzing each phase separately suggested that the difference in recorded and model maximum amplitudes was entirely attributable to the gregarious locusts $\left(F_{(1,31)}=13.4\right.$; $p<0.001)$, with no significant differences between these values among solitarious locusts $\left(F_{(1,29)}=0.87 ; p=0.36\right)$.

The maximum amplitudes of FETi responses predicted by the model did not differ with $l /|v|$ for either phase, which corresponded to the recorded responses of FETi. The only difference between the model and recorded data was that the recorded response of FETi in gregarious locusts was consistently greater than would be predicted.

We electrically stimulated DCMD to produce trains of PSPs at regular intervals to analyze the possibility that the discrepancy between model and recorded values could be explained by shortterm synaptic plasticity (Fig. 7). Brief (0.05 ms) electrical stimulation of the cervical connectives produced spikes that were similar to those of DCMD and evoked PSPs in FETi that were indistinguishable in amplitude from those elicited from visually stimulated DCMD spikes (paired samples $t$ test, $t_{9}=-0.827 ; p=$ 0.43 ; data taken from both phases). The latency between the spike evoked by the electrical stimulus in the pro-mesothoracic connectives and the PSP was also the same as that between visually evoked DCMD spikes and the PSP $\left(t_{9}=1.741 ; p=0.116\right.$; mean difference between electrically and visually elicited spikes, 0.06 $\mathrm{ms})$. The similarity in PSP latency and amplitude between electrically and visually elicited PSPs indicate that both were driven by DCMD only. Repeated electrical stimulation at $30 \mathrm{~ms}$ intervals evoked a train of PSPs, which showed only a small amount of 

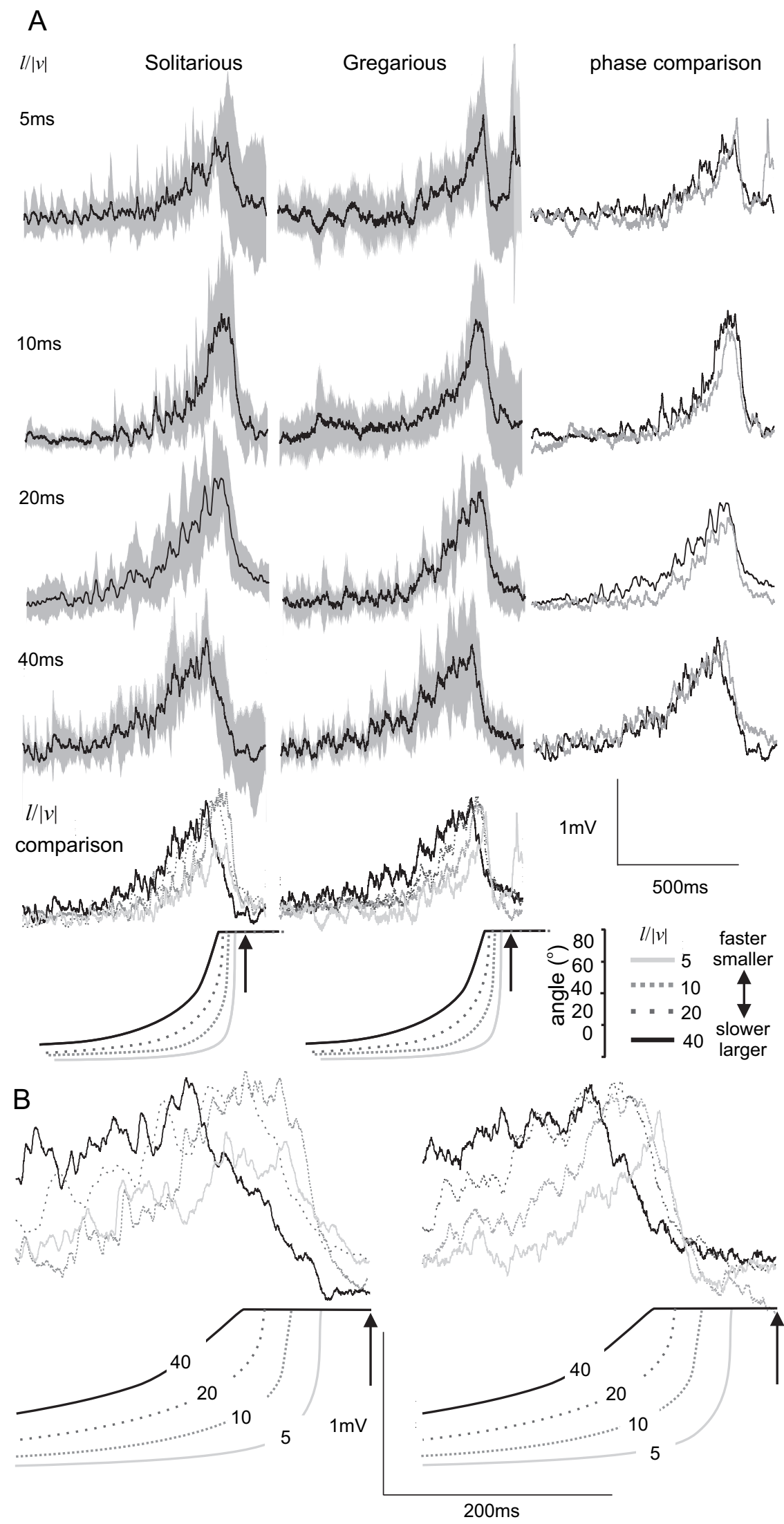

Figure 5. A, Average (black lines) \pm SD (gray bands) compound PSPs elicited by DCMD in FETi after stimulation of DCMD by looming objects of different $/ /|v|(n=8$ solitarious and 9 gregarious locusts). The right column overlays the mean responses of the two phases to each stimulus. Beneath are overlays of the mean responses to objects of different $|/| v \mid$. $\boldsymbol{B}$, Overlays of the mean responses to objects of different $I /|v|$ at an expanded time scale showing mean times of peak firing, as indicated by the numbers on the curves subtended by the expanding objects. The arrows indicate the time of collision. summation (Fig. 7A). Comparison of successive PSP amplitudes showed that the synapse had facilitated (Fig. $7 B, C$ ) and that the degree of facilitation differed between phases (repeated-measures ANOVA on percentage change in PSP amplitude, $F_{(8,64)}=11.681, p<0.001$; interaction between relative amplitude and phase $F_{(8,64)}=2.984, p=0.007$; only the first nine PSPs were included in the analysis to allow enough degrees of freedom). Gregarious locusts showed much stronger facilitation than solitarious locusts, with the second pulse in the train being $15 \pm$ $5.2 \%$ larger than the first (one-sample $t$ test against a normalized first PSP amplitude of $1 ; t_{4}=6.467 ; p=0.003$ ) (Fig. $7 C$ ). Successive PSPs in the train further increased in amplitude, becoming $32 \pm$ 9.1\% larger than the first PSP by the sixth in the series and then maintaining an approximately constant amplitude for the rest of the series. Solitarious locusts showed much more variable levels of facilitation, and indeed one of the five tested animals displayed a marginal depression of synaptic strength over the course of the stimulus train. The second PSP was only $5 \pm 10 \%$ greater than the first PSP $\left(t_{4}=\right.$ 1.094; $p=0$. 335; not significant) and reached a maximum $13.8 \pm 13.5 \%$ increase in amplitude by the fourth PSP in the sequence. By the eighth PSP in the sequence, amplitude had begun to decline again (Fig. $7 C$ ).

The time of maximum response in FETi The time at which the maximum amplitude of the compound PSP occurred in FETi was nonlinearly related to $l /|v|$ (Fig. $8 A, B)$, although the time of peak firing in DCMD is linear with respect to $l /|v|$ (Fig. $3 C)$. In other words, the DCMD-FETi synapse transformed the relationship between $l /|v|$ and the time of maximum firing rate/ peak depolarization. Furthermore, whereas there was no difference in the time of peak firing in DCMD between solitarious and gregarious locusts (Fig. $3 C$ ), in FETi, the timing of maximum PSP amplitude with respect to $l /|v|$ differed significantly between phases (Mann-Whitney test comparing the exponential coefficients of curves with the equation $y(x)=y_{0}$ $+a \times e^{-b x}$ fitted to the data in Fig. 6A: $y_{0}$, $Z=-2.1, p=0.036$; $a, Z=-1.9, p=$ 0.059 ; b, $Z=-2, p=0.046$; $n=16$ ). In solitarious locusts, the times of peak PSP amplitude with changing $l /|v|$ were nearer to the linear relationship found in DCMD (median exponential term, $\mathrm{b}=0.03$, where an exponential term of 0 would give a straight line) (Fig. $8 A, B$ ). In gregarious 
locusts, however, the relationship between time of FETi maximum and $l /|v|$ was more strongly curved (median exponential term, $\mathrm{b}=0.06)$. The model of FETi compound PSPs exhibited the same linear relationship between the time of FETi maximum and $l /|v|$ as the underlying DCMD response in both phases. Hence, the gradients of linear regressions of the time of maximum simulated FETi response against time of maximum DCMD response did not differ significantly from 1 (one-sample $t$ test; $t_{16}=1.66 ; n=17$ ).

Comparing the predicted time of FETi maximum amplitude from the models with the measured experimental values revealed that in gregarious locusts, the maximum FETi response occurred earlier than would be predicted when $l /|v|<40 \mathrm{~ms}$ (Fig. 8C-E). Recordings at an expanded time scale (Fig. 8C) show that the time of PSP maximum (black arrows) occurred well before the maximum predicted by the model (gray arrows) in a gregarious locust for stimuli of $l /|v|=5$ or $10 \mathrm{~ms}$. In a solitarious locust, the recorded PSP maxima occurred much closer to the predicted time. Both phases show a near coincident time of PSP maximum for $l /|v|=40 \mathrm{~ms}$ (Fig. $8 C$, bottom pair of recordings). The data from all recordings are shown in Figure $8, D$ (all data) and $E$ (medians and interquartile ranges), with the diagonal lines showing where the measured and predicted values would be the same. In gregarious locusts, when $l /|v|<40 \mathrm{~ms}$, the measured times of FETi maximum fell consistently below this line, indicating that the peak occurred earlier than predicted (Fig. $8 D, E$, gray symbols) (the mean measured time of FETi maximum minus the predicted value was $12 \mathrm{~ms}, t$ test against an expected difference of $0 \mathrm{~ms} ; t_{34}=-2.69$; $p=0.01$ ). When $l /|v|=40 \mathrm{~ms}$, however, there was no significant difference between the measured and predicted values of time of FETi maximum $\left(t_{18}=0.63 ; p=0.54\right.$; mean difference, $\left.0.7 \mathrm{~ms}\right)$. In contrast, in solitarious locusts, most recorded times of FETi maximum amplitude were near those predicted by the model, particularly at lower values of $l /|v|$ (Fig. $8 D, E$, black symbols). The mean difference between the measured and predicted times of maximum FETi response did not differ significantly from zero for any value of $l /|v|$ (by comparison with the gregarious data above: for $l /|v|<40 \mathrm{~ms}$, the mean difference was $0.04 \mathrm{~ms}, t_{38}=-0.28, p=0.97$; for $l /|v|=40 \mathrm{~ms}, t_{27}=0.97, p=0.34$ ). Although solitarious locusts did not display any systematic shift in the time of maximum PSP amplitude in the same way as gregarious locusts, they exhibited a greater variance in the residual difference between measured and predicted times of peak amplitude than gregarious locusts (Fig. $8 F)\left(\right.$ ANOVA; $\left.F_{(1,117)}=4.15 ; p<0.044\right)$. The differences between measured and predicted values also increased systematically with $l /|v|$ for both phases (Fig. $8 F$ ) (ANOVA; $\left.F_{(1,117)}=6.73 ; p<0.011\right)$.
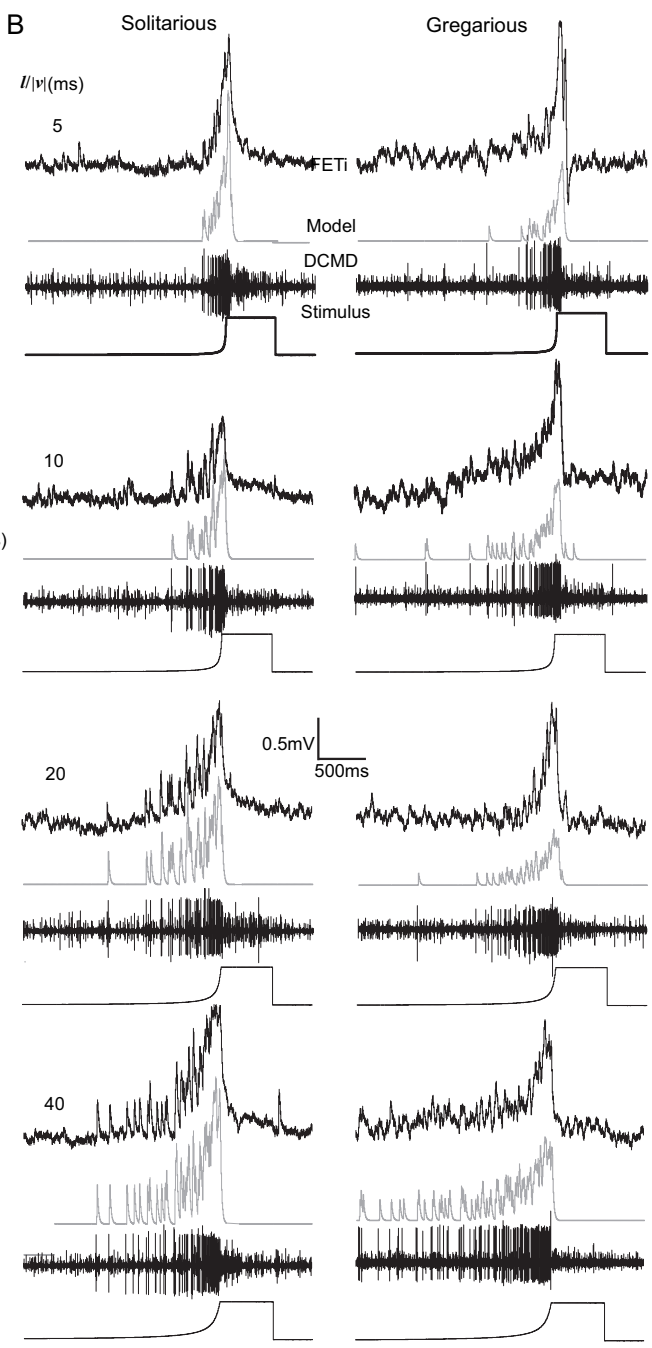

Figure 6. The maximum amplitudes of compound PSPs in FETi were always similar regardless of phase or $/ /|v| \cdot \boldsymbol{A}$, Regressions fitted to the maximum FETi PSP amplitudes in solitarious (black) and gregarious locusts over a range of different values of $/ /|v| \cdot \boldsymbol{B}$ Recordings of DCMD and FETi from a solitarious (left column) and gregarious (right column) locust together with model wavethe maximum compound PSP amplitude predicated by the model with the unitary PSP template used to generate it. $D$, Median and $50 \%$ data ranges for the data in $\boldsymbol{C}$ by phase and for each value of $I /|v|$. $\boldsymbol{E}$, Means and SDs of maximum compound PSP amplitudes compared with expected amplitudes obtained from the model.

\section{Effect of DCMD habituation on FETi response}

DCMD habituated during repeated stimulation with a looming object of $l /|v|=20 \mathrm{~ms}$ at $1 \mathrm{~min}$ intervals (data for peak firing rate are shown in Fig. 9A). Habituation was considerably stronger in solitarious phase locusts, in agreement with our previous study (Matheson et al., 2004). By the seventh approach (the last stimulus in which all solitarious locusts produced two or more spikes to the stimulus), peak DCMD firing rate had decreased, on average, by $66 \%$ in solitarious locusts but only by $34 \%$ in gregarious ( $t$ test; $\left.t_{15}=4.19 ; p<0.001\right)$. The data were fitted to a simple exponential decay curve of the form $y(x)=y_{0}+a \times e^{-b x}$. In solitarious locusts, the asymptote was at 70 spikes $\mathrm{s}^{-1}$, whereas in gregarious locusts, it was over twice as high, at 157 spikes s $^{-1}$ (Fig. 9A).

The maximum amplitude of the compound PSP decreased on repeated visual stimulation, in a pattern that was similar to the change in DCMD response (Fig. 9B). The maximum amplitude of the compound PSP decreased on repeated visual stimulation, in a similar way to the change in DCMD (Fig. 9B). The maximum 
A

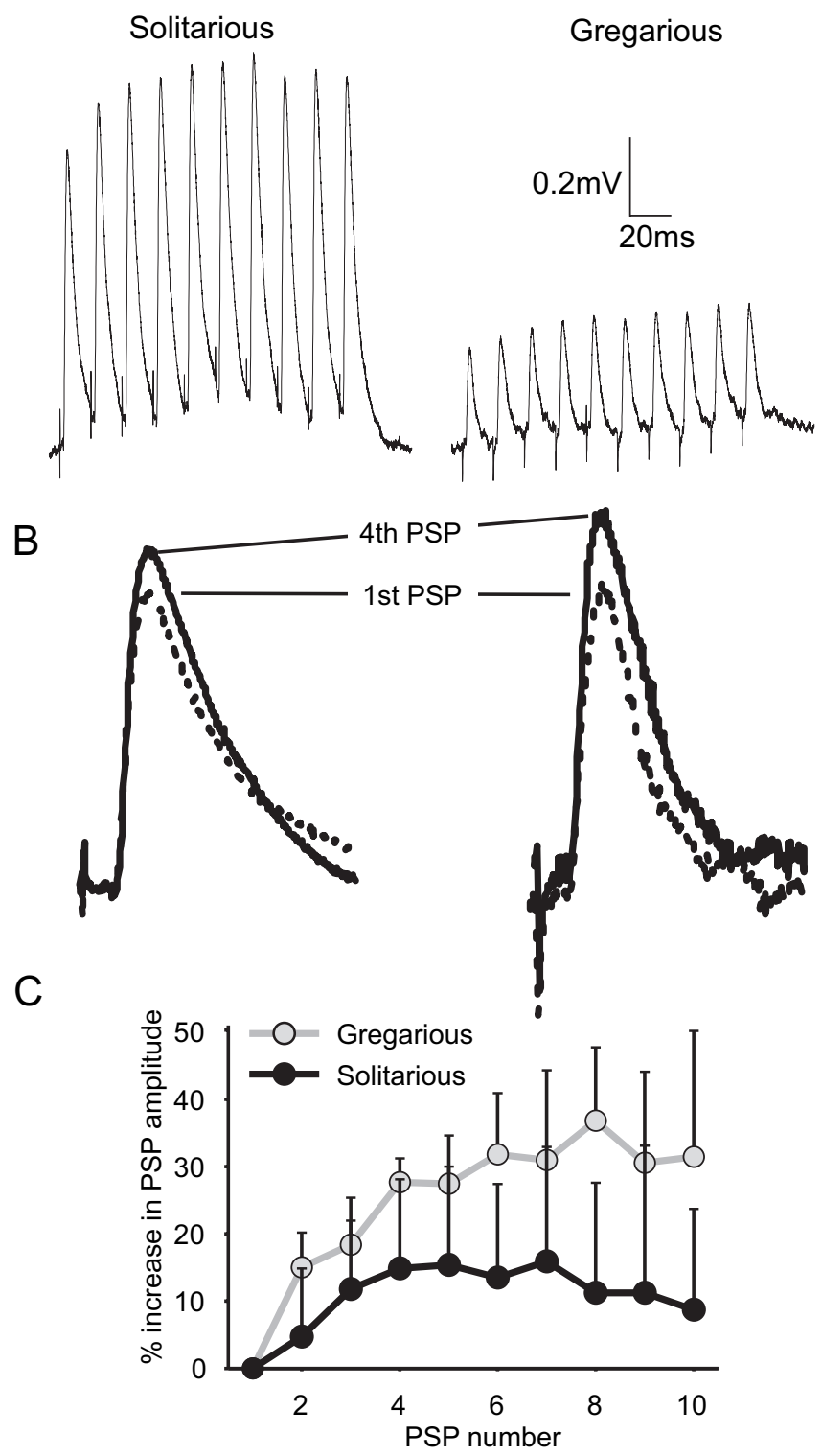

Figure 7. The DCMD-FETi synapse facilitates on repeated stimulation. $\boldsymbol{A}$, Intracellular recordings from FETi of representative solitarious (left) and gregarious (right) locusts in which DCMD has been driven by electrical stimulation of the ipsilateral cervical connective at $30 \mathrm{~ms}$ intervals. $\boldsymbol{B}$, The first PSPs in each sequence (dotted lines) rescaled to the same amplitude and overlaid with the corresponding fourth PSP to show the relative increases in amplitude during the stimulus train. $C$, The mean percentage increase in PSP amplitude relative to the first PSP in gregarious (gray) and solitarious (black) locusts over the course of 10 stimulus pulses $(n=5$ animals of each phase).

amplitude of the compound PSP in FETi was similar for both solitarious and gregarious locusts during the first approach $\left(1.9 \pm 0.47\right.$ and $1.7 \pm 0.53 \mathrm{mV}$, respectively; $t$ test; $t_{15}=0.786$; $p=0.44)$, but by the fourth approach there was a clear difference in the amplitudes of the compound PSPs between phases (Fig. $8 B)\left(0.9 \pm 0.32\right.$ and $1.4 \pm 0.39 \mathrm{mV} ; t$ test; $t_{15}=-3.168 ; p=$ $0.006)$. By the seventh approach, the frequency of DCMD spikes in solitarious locusts was often so low that the response in FETi had decomposed into a series of individual nonsummating PSPs (Fig. 10A), so that the "compound" response amplitude was similar to that of individual PSPs (Fig. 9B, dark gray band). The mean compound PSP amplitude by the seventh approach was $1.16 \pm$
$0.42 \mathrm{mV}$ in gregarious locusts and only $0.72 \pm 0.33 \mathrm{mV}$ in solitarious locusts ( $t$ test; $t_{15}=-2.404 ; p=0.03$ ).

When visual stimuli are repeated at intervals shorter than 1 min, DCMD responses of both solitarious and gregarious locusts habituate even more strongly, but responses in gregarious locusts always exceed those in solitarious locusts for a given repetition rate (data not shown). We have not examined the response of FETi to these higher stimulation frequencies.

The maximum compound PSP amplitude was constant in nonhabituated locusts regardless of phase or $l /|v|$, but there was a linear relationship between the decrease in peak DCMD firing rate as it habituated and the maximum amplitude of the compound PSP, which differed between phases (Fig. 9C). Linear regressions were performed on the data for each animal, and the resulting gradients were compared between phases. In solitarious locusts, the compound PSP maximum amplitude depended on the firing rate as follows: $0.01 \pm 0.004 \mathrm{mV} /$ spikes. $^{-1}$, but in gregarious locusts, the gradient was shallower at $0.004 \pm 0.004$ $\mathrm{mV} /$ spikes.s $^{-1}$. The reduction in the frequency of DCMD spikes during habituation therefore had a stronger effect on compound PSP amplitude in solitarious locusts than on gregarious locusts $(t$ test; $\left.t_{15}=15.0 ; p=0.005\right)$. The amplitudes of single PSPs elicited by the first DCMD spike of each stimulus remained the same over the course of the habituation experiments and showed no evidence of themselves habituating (ANOVA; $F_{(9,145)}=0.75 ; p=$ $0.63)$.

The DCMD spike trains obtained during the habituation experiments were convolved with PSP templates and compared between phases. As before, the model was scaled to produce voltage values by matching the sizes of the first recorded and simulated single PSPs. In solitarious locusts (Fig. 10A-C), the model predicted smaller maximum compound PSP amplitudes than those recorded in FETi during the first two approaches (model prediction, $1.58 \pm 0.47 \mathrm{mV}$ compared with recorded maximum of $1.92 \pm 0.47$; a $21 \%$ difference). By the third approach, however, the model had converged with the real data (model prediction, $0.96 \pm 0.53 \mathrm{mV}$ compared with recorded maximum $1.02 \pm$ $0.42 \mathrm{mV}$; a $6 \%$ difference). In gregarious locusts (Fig. 10A-C), there was a much greater disparity between the maximum amplitudes predicted by the model and the larger measured values over the entire series of approaches. During the first approach, there was a $52 \%$ difference between the predicted and the recorded maximum amplitudes $(1.13 \pm 0.45$ against $1.73 \pm 0.53 \mathrm{mV})$. Even by the seventh approach, the difference between predicted and observed values was still $45 \%$ ( $0.80 \pm 0.32$ compared with $1.16 \pm 0.42 \mathrm{mV})$. There was no evidence that observed and predicted maximum compound PSP amplitude values would have converged with further visual stimulation, because the asymptotes of the curves fitted in Figure $10 \mathrm{~B}$ were $1.11 \mathrm{mV}$ for the measured data and $0.74 \mathrm{mV}$ for the model data, indicating a consistent $50 \%$ difference. We conclude that the facilitation of the DCMD-FETi synapse that occurs in gregarious locusts does not itself habituate.

The time of the peak DCMD firing rate relative to collision became more variable as DCMD habituated and was therefore more likely to occur earlier in the approach (data not shown). This tendency was particularly marked in solitarious locusts, as reported previously by Matheson et al. (2004). This in turn affected the time of the maximum amplitude of the compound PSP, but there was no evidence for any other systematic change in the time of PSP maximum amplitude that did not derive from the underlying variability in DCMD (ANOVA on the magnitude of the residuals of regressions of time of FETi maximum against 
time of peak DCMD firing; approach $F_{(9,}$, 131) $=0.75, p=0.66$; phase $F_{(1,131)}=0.85$, $p=0.36$ ).

\section{Discussion}

Phase change is a naturally occurring polyphenism that causes extensive and wideranging changes to most physiological systems of desert locusts, including their sensory and motor systems. We characterized homeostatic plasticity acting at an identified sensory-motor synapse that maintains a constant amplitude of response in the face of these otherwise widespread changes. The response of DCMD changes with the size or speed of looming stimuli and was less sensitive in solitarious locusts than gregarious locusts (33-40\% lower peak firing rates and $30-70 \%$ fewer spikes), but the single PSPs elicited in solitarious FETi were twice the amplitude of those in gregarious locusts. Looming stimuli, however, elicited compound PSPs in FETi that had the same amplitude in both phases regardless of the velocity or size of the stimulus. The timing of the compound PSP maximum in FETi was nonlinearly advanced relative to that predicted by the response of DCMD, particularly so in gregarious locusts. In the present study, responses were only elicited after $8-10 \mathrm{~min}$ intervals, ensuring that DCMD was in a fully dishabituated state, whereas previously (Matheson et al., 2004), stimuli were presented at $1 \mathrm{~min}$ intervals, leading to partial DCMD habituation particularly in solitarious locusts.

Previous studies of homeostatic plasticity have primarily followed the consequences of experimentally imposed disruptions of neuronal function. For example, genetic manipulation (Davis et al., 1997; Sandrock et al., 1997; Davis and Goodman, 1998; Guan et al., 2005), physical isolation of parts of the nervous system (Turrigiano et al., 1995; Thoby-Brisson and Simmers, 1998), pharmacological treatments (Berg and Hall, 1975; Ramakers et al., 1990; Turrigiano et al., 1998), or sensory deprivation during development (Desai et al., 2002). Only the scaling of synaptic inputs over the course of normal postnatal development shows a similar naturally occurring homeostatic plasticity (Desai et al., 2002). Bucher et al. (2005) demonstrate that there is homeostatic regulation of whole-network function over a developmental timescale in the lobster pyloric network but do not identify the specific underlying homeostatic processes.

Homeostatic plasticity may lead to altered signaling costs, as shown by robustness in neuronal signaling in the face of the loss of a delayed rectifier potassium channel in Drosophila photoreceptors (Vahasoyrinki et al., 2006). Because the cost of propagating action potentials is generally greater than the cost of synaptic transmission (Laughlin et al., 1998), the DCMD-
FETi pathway is likely to require less energy in solitarious locusts. This would occur at the cost of a coarser and less precise temporal tuning of peak activity in DCMD (Matheson et al., 2004). If this pattern were repeated throughout the CNS, it could translate into a considerably reduced metabolic operating cost in solitarious locusts, which are less active, show reduced adipokinetic responses, and presumably have lower metabolic rates than gregarious locusts (Pener, 1991; Pener et al., 1997). The homeostatic plasticity in the DCMD-FETi pathway of locusts may compensate for changes in neuronal excitability, resulting from widespread phase-related changes in the CNS levels of many neurochemicals (Rogers et al., 2004). Of greater significance, however, may be the differences 
A

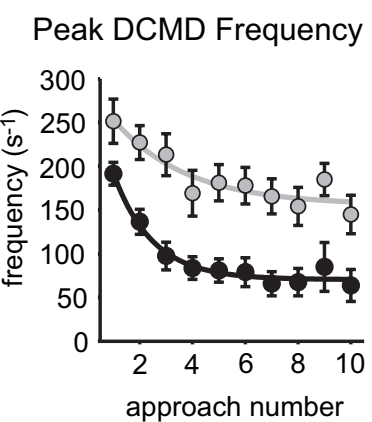

B Maximum FETi Amplitude

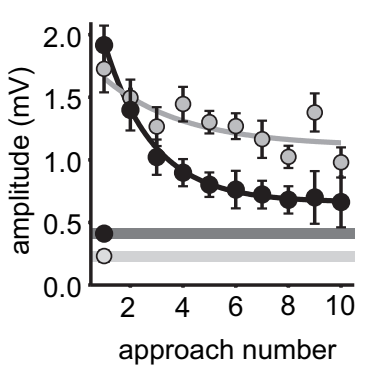

C

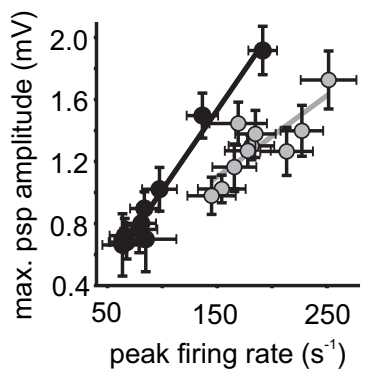

Figure 9. Habituation of DCMD is accompanied by habituation of the FETi compound PSP. $A$, Peak firing frequency in DCMD habituated on repeated stimulation by a looming object of $|/| v \mid=20 \mathrm{~ms}$ at $1 \mathrm{~min}$ intervals. The habituation was stronger in solitarious (black) than gregarious (gray) locusts. $\boldsymbol{B}$, The maximum amplitude of the compound PSP in FETi also decreased on repeated stimulation. In solitarious locusts, the amplitudes diminished within 10 stimuli to nearly the same amplitudes as single PSPs (dark gray band). The light gray band indicates the equivalent amplitudes of single PSPs in gregarious locusts. $\boldsymbol{C}$, The relationship between peak compound PSP amplitude and peak DCMD firing rate differed between phases. All data are means \pm SEM.
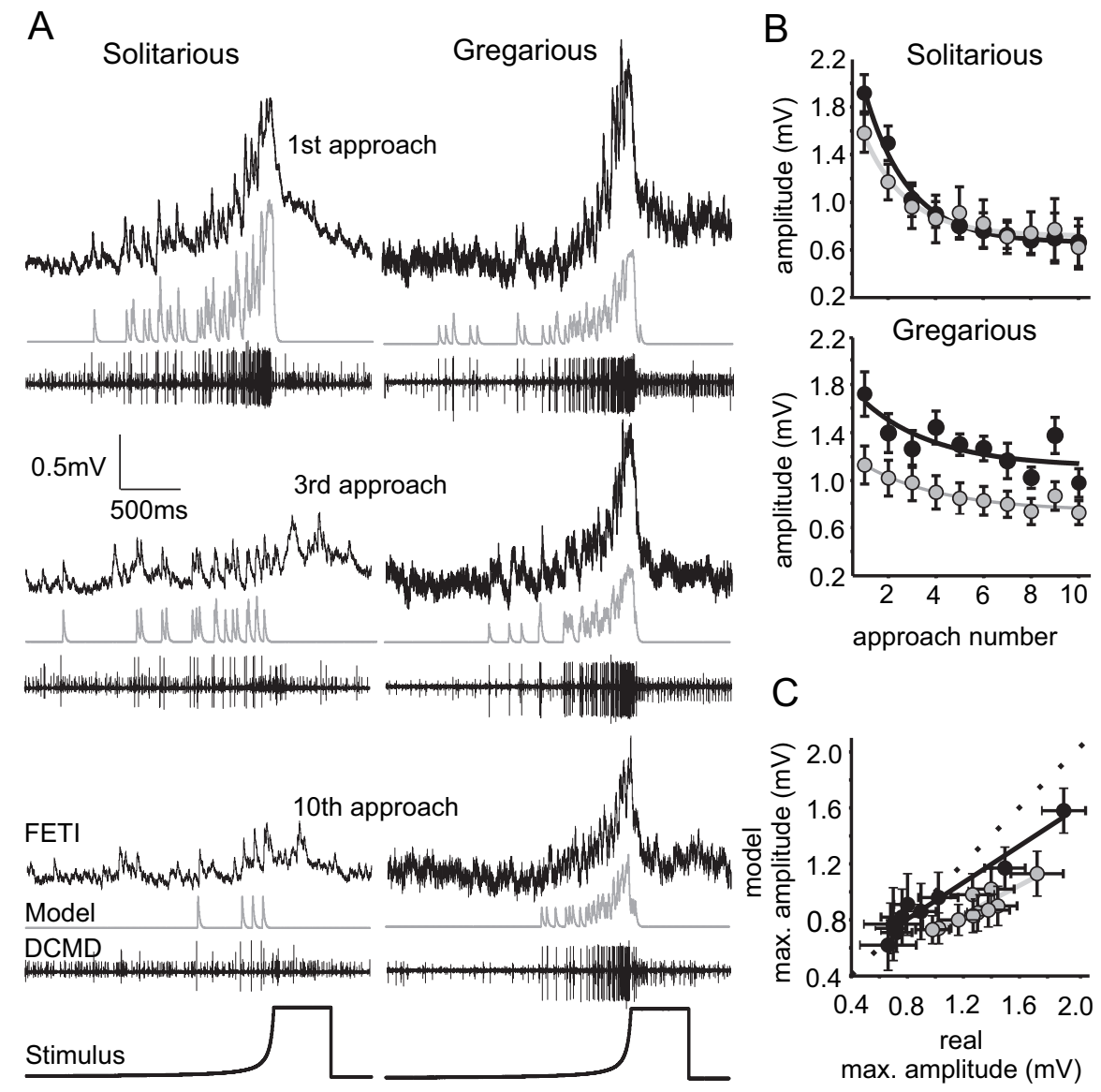

Figure 10. $\quad \boldsymbol{A}$, Examples of FETi and DCMD recordings together with model waveforms from solitarious (left column) and gregarious (right column) locusts taken over the course of DCMD habituation. $\boldsymbol{B}$, Measured (black) and predicted (gray) maximum PSP amplitudes showed a rapid convergence during habituation in solitarious locusts (left) but remained different throughout DCMD habituation in gregarious locusts (right). C, The relationships between observed and predicted maximum compound PSP amplitudes differed between solitarious (black) and gregarious (gray) locusts. All data are means \pm SEM.

in timing and variability of the response that arise as a direct consequence of the homeostatic mechanism. This tuning may adapt the locust to the different behavioral requirements of each phase.
Three components of homeostatic plasticity at the FETi-DCMD synapse Compound PSPs modeled by convolving experimentally constrained PSP templates with DCMD spike trains matched the measured values well in solitarious locusts but underestimated them by $33 \%$ in gregarious locusts. The greater maximum firing frequency of DCMD in gregarious locusts only partially offset the decreased size of individual PSPs, and thus a third process must be involved in regulating the maximum amplitude of the compound PSP between phases. The disparity in amplitude increased progressively over the course of the stimulus. A non-DCMD synaptic input to FETi, such as from a synaptic input from other visual interneurons or from indirect feedforward inputs from DCMD, would have to produce a smooth, slowly rising depolarization in FETi over a long period $(>200 \mathrm{~ms})$. Our electrical stimulation data show that the difference can be accounted for by facilitation of the DCMD-FETi synapse during repeated DCMD spiking in gregarious locusts, which was not accounted for in the model. Such short-term synaptic plasticity is well documented in insects (Burrows et al., 1989; Parker, 1995; Niven and Burrows, 2003). In solitarious locusts, the similarity between predicted and measured compound PSP amplitudes suggests that there was little facilitation during looming stimuli, in accordance with our electrical stimulation experimental data. In summary, the constancy of the overall drive onto FETi during a looming stimulus arises from homeostatically balanced contributions from DCMD firing rates, synaptic strength, and a third process, short-term synaptic plasticity

LGMD-DCMD displays a linear relationship between the time of peak firing frequency relative to collision and the size/ velocity $(l /|v|)$ of an approaching object (Hatsopoulos et al., 1995; Gabbiani et al., $1999,2001,2002)$ that is invariant between locust phases (Matheson et al., 2004). This relationship was transformed by the DCMD-FETi synapse so that there was a nonlinear relationship between $l /|v|$ and the time of maximum PSP amplitude, and a clear difference between solitarious and gregarious locusts. In solitarious locusts, the nonlinearity was small, but in gregarious locusts, it was much greater so that the time of peak depolarization occurred markedly earlier for smaller values of $l /|v|$. To achieve this synaptic facilitation earlier in the response would have to be followed by synaptic depression (Markram et al., 1998; Thomson, 2000) or intermittent failure. Competing processes of synaptic facilitation and depression can act as a bandpass filter, 
selectively emphasizing certain frequencies in a burst of spikes (Sen et al., 1996; Jorge-Rivera et al., 1998; Izhikevich et al., 2003). This may allow different postsynaptic neurons to respond preferentially to different parts of spike train or to favor stronger responses in some postsynaptic targets. At the DCMD-FETi synapse, such an interaction may shape the time at which the compound PSP maximum occurs. The same process may also reduce variability in the time of peak depolarization. When stimulated by a looming object, DCMD produces a burst of spikes with an escalating frequency, but it can occasionally produce short highfrequency bursts earlier in the approach. Synaptic facilitation will tend to filter out earlier fluctuations in the firing rate of DCMD (when evoked PSPs are small) and emphasize the later sustained high-frequency spikes (when the largest individual PSP are evoked). This may act to counteract noise in the sensory signal (Krahe and Gabbiani, 2004) and variation in the measured time of peak FETi response amplitude relative to that predicted by the model was less in gregarious than in solitarious locusts. In solitarious locusts, there was less synaptic facilitation and possibly depression and fewer DCMD spikes so that the time of peak compound PSP amplitude was more variable, potentially making the behavioral response more unpredictable to predators. Determining the full relationship between DCMD firing rate and FETi synaptic plasticity, and thus establishing the balance between facilitation and depression, will be an important step in fully understanding the shaping of the response to looming stimuli.

\section{Behavioral consequences}

The amplitude of the compound PSP produced by DCMD in FETi was always below spiking threshold in both phases and thus could not initiate a behavioral response in the absence of another synaptic drive. Jumping in locusts requires a complex motor pattern (Heitler and Burrows, 1977a,b; Burrows, 1995; Santer et al., 2005b) with energy stored in elastic elements in the leg during a prolonged cocontraction between extensor and flexor tibiae muscles. This process is aided by a monosynaptic excitatory connection between FETi and the flexor tibiae motoneurons (Hoyle and Burrows, 1973; Niven and Burrows, 2003). A jump is triggered when the flexor tibiae motoneurons stop firing, allowing rapid extension of the tibia. This entire process takes several hundred milliseconds, and thus DCMD can have little role in initiating the process if escape is to precede collision. DCMD could, however, have a significant function in an already cocontracting animal by adding to the excitation in FETi, controlling the timing of kick release (Niven and Burrows, 2003), or by possibly providing a shunting inhibition to the FETi-flexor synapses that would assist in releasing a kick. Our data suggest that the strength of any such effects will be the same in both phases, although the timing at which they occur will differ.

The visual environment of gregarious locusts is, by definition, dominated by the presence of other locusts, which provide a constant source of small fast-moving objects ( $\operatorname{small} l /|v|)$. Gregarious locusts are more visible to predators than cryptic solitarious locusts, but once detected, they are protected by numbers. Differences in the sensory-motor pathway from DCMD provide gregarious locusts with an earlier and more temporally consistent response to approaching objects, mostly other locusts, but give solitarious locusts a more variable later-acting escape response to predators.

\section{References}

Berg DK, Hall ZW (1975) Increased extrajunctional acetylcholine sensitivity produced by chronic post-synaptic neuromuscular blockade. J Physiol (Lond) 244:659-676.
Bucher D, Prinz AA, Marder E (2005) Animal-to-animal variability in motor pattern production in adults and during growth. J Neurosci 25:1611-1619.

Burrows M (1995) Motor patterns during kicking movements in the locust. J Comp Physiol A Neuroethol Sens Neural Behav Physiol A 176:289-305.

Burrows M, Matheson T (1994) A presynaptic gain control mechanism among sensory neurons of a locust leg proprioceptor. J Neurosci 14:272-282.

Burrows M, Rowell CHF (1973) Connections between descending visual interneurons and metathoracic motoneurons in the locust. J Comp Physiol 85:221-234.

Burrows M, Watson AHD, Brunn DE (1989) Physiological and ultrastructural characterization of a central synaptic connection between identified motor neurons in the locust. Eur J Neurosci 1:111-126.

Davis GW, Goodman CS (1998) Genetic analysis of synaptic development and plasticity: homeostatic regulation of synaptic efficacy. Curr Opin Neurobiol 8:149-156.

Davis GW, Schuster CM, Goodman CS (1997) Genetic analysis of the mechanisms controlling target selection: target-derived fasciclin II regulates the pattern of synapse formation. Neuron 19:561-573.

Desai NS, Cudmore RH, Nelson SB, Turrigiano GG (2002) Critical periods for experience-dependent synaptic scaling in visual cortex. Nat Neurosci 5:783-789.

Gabbiani F, Krapp HG, Laurent G (1999) Computation of object approach by a wide-field, motion-sensitive neuron. J Neurosci 19:1122-1141.

Gabbiani F, Mo CH, Laurent G (2001) Invariance of angular threshold computation in a wide-field looming-sensitive neuron. J Neurosci 21:314-329.

Gabbiani F, Krapp HG, Koch C, Laurent G (2002) Multiplicative computation in a visual neuron sensitive to looming. Nature 420:320-324.

Guan Z, Saraswati S, Adolfsen B, Littleton JT (2005) Genome-wide transcriptional changes associated with enhanced activity in the Drosophila nervous system. Neuron 48:91-107.

Hatsopoulos N, Gabbiani F, Laurent G (1995) Elementary computation of object approach by a wide-field visual neuron. Science 270:1000-1003.

Heitler WJ, Burrows M (1977a) The locust jump. 1. The motor programme. J Exp Biol 66:203-219.

Heitler WJ, Burrows M (1977b) The locust jump. 2. Neural circuits of the motor programme. J Exp Biol 66:221-241.

Horridge GA (1978) The separation of visual axes in apposition compound eyes. Philos Trans R Soc Lond B Biol Sci 285:1-59.

Howard J (1981) Temporal resolving power of the photoreceptors of Locusta migratoria. J Comp Physiol 144:61-66.

Hoyle G, Burrows M (1973) Neural mechanisms underlying behaviour in the locust Schistocerca gregaria. I. Physiology of identified motoneurons in the metathoracic ganglion. J Neurobiol 4:3-41.

Izhikevich EM, Desai NS, Walcott EC, Hoppensteadt FC (2003) Bursts as a unit of neural information: selective communication via resonance. Trends Neurosci 26:161-167.

Jackson MB, Konnerth A, Augustine G (1991) Action potential broadening and frequency-dependent facilitation of calcium signals in pituitary nerve terminals. Proc Natl Acad Sci USA 88:380-384.

Jorge-Rivera JC, Sen K, Birmingham JT, Abbott LF, Marder E (1998) Temporal dynamics of convergent modulation at a crustacean neuromuscular junction. J Neurophysiol 80:2559-2570.

Krahe R, Gabbiani F (2004) Burst firing in sensory systems. Nat Rev Neurosci 5:13-23.

Laughlin SB, van Steveninck RRD, Anderson JC (1998) The metabolic cost of neural information. Nat Neurosci 1:36-41.

Markram H, Wang Y, Tsodyks M (1998) Differential signaling via the same axon of neocortical pyramidal neurons. Proc Natl Acad Sci USA 95:5323-5328.

Matheson T, Rogers SM, Krapp HG (2004) Plasticity in the visual system is correlated with a change in lifestyle of solitarious and gregarious locusts. J Neurophysiol 91:1-12.

Niven JE, Burrows M (2003) Spike width reduction modifies the dynamics of short-term depression at a central synapse in the locust. J Neurosci 23:7461-7469.

Nusbaum MP, El Manira A, Gossard JP, Rossignol S (1997) Presynaptic mechanisms during rhythmic activity in vertebrates and invertebrates. In: Neurons, networks and motor behavior (Stein PSG, Grillner S, Selverston AI, Stuart DG, eds), pp 237-253. Cambridge, MA: MIT. 
O'Shea M, Williams JLD (1974) The anatomy and output connection of a locust visual interneurone: the lobula giant movement detector (LGMD) neurone. J Comp Physiol 91:257-266.

Parker D (1995) Depression of synaptic connections between identified motor neurons in the locust. J Neurophysiol 74:529-538.

Pearson KG, Goodman CS (1979) Correlation of variability in structure with variability in synaptic connections of an identified interneurone in locusts. J Comp Neurol 184:141-166.

Pearson KG, Heitler WJ, Steeves JD (1980) Triggering of locust jump by multimodal inhibitory interneurons. J Neurophysiol 43:257-277.

Pener MP (1991) Locust phase polymorphism and its endocrine relations. Advances Insect Physiol 23:1-79.

Pener MP, Ayali A, Golenser E (1997) Adipokinetic hormone and flight fuel related characteristics of density-dependent locust phase polymorphism: a review. Comp Biochem Physiol B 117:513-524.

Ramakers GJA, Corner MA, Habets AMMC (1990) Development in the absence of spontaneous bioelectric activity results in increased stereotyped burst firing in cultures of dissociated cerebral-cortex. Exp Brain Res 79:157-166.

Rind FC, Simmons PJ (1992) Orthopteran DCMD neuron-a reevaluation of responses to moving-objects. I. Selective responses to approaching objects. J Neurophysiol 68:1654-1666.

Roessingh P, Simpson SJ, James S (1993) Analysis of phase-related changes in behaviour of desert locust nymphs. Proc R Soc Lond B Biol Sci 252:43-49.

Rogers SM, Matheson T, Sasaki K, Kendrick K, Simpson SJ, Burrows M (2004) Substantial changes in central nervous system neurotransmitters and neuromodulators accompany phase change in the locust. J Exp Biol 207:3603-3617.

Sandrock AW, Dryer SE, Rosen KM, Gozani SN, Kramer R, Theill LE, Fischbach GD (1997) Maintenance of acetylcholine receptor number by neuregulins at the neuromuscular junction in vivo. Science 276:599-603.

Santer RD, Simmons PJ, Rind FC (2005a) Gliding behaviour elicited by lateral looming stimuli in flying locusts. J Comp Physiol A Neuroethol Sens Neural Behav Physiol 191:61-73.

Santer RD, Yamawaki Y, Rind FC, Simmons PJ (2005b) Motor activity and trajectory control during escape jumping in the locust Locusta migratoria. J Comp Physiol A Neuroethol Sens Neural Behav Physiol 191:965-975.

Santer RD, Rind FC, Stafford R, Simmons PJ (2006) Role of an identified looming-sensitive neuron in triggering a flying locust's escape. J Neurophysiol 95:3391-3400.

Schlotterer GR (1977) Response of the locust descending movement detec- tor neuron to rapidly approaching and withdrawing visual stimuli. Can J Zool 55:1372-1376.

Sen K, Jorge-Rivera JC, Marder E, Abbott LF (1996) Decoding synapses. J Neurosci 16:6307-6318.

Simmons PJ (1980) Connexions between a movement-detecting visual interneurone and flight motoneurones of a locust. J Exp Biol 86:87-97.

Simmons PJ, Rind FC (1992) Orthopteran DCMD neuron-a reevaluation of responses to moving-objects. II. Critical cues for detecting approaching objects. J Neurophysiol 68:1667-1682.

Simpson SJ, McCaffery AR, Hagele BF (1999) A behavioural analysis of phase change in the desert locust. Biol Rev 74:461-480.

Thoby-Brisson M, Simmers J (1998) Neuromodulatory inputs maintain expression of a lobster motor pattern-generating network in a modulationdependent state: evidence from long-term decentralization in vitro. J Neurosci 18:2212-2225.

Thomson AM (2000) Molecular frequency filters at central synapses. Prog Neurobiol 62:159-196.

Turrigiano GG (1999) Homeostatic plasticity in neuronal networks: the more things change, the more they stay the same. Trends Neurosci 22:221-227.

Turrigiano GG, Nelson SB (2000) Hebb and homeostasis in neuronal plasticity. Curr Opin Neurobiol 10:358-364.

Turrigiano GG, Nelson SB (2004) Homeostatic plasticity in the developing nervous system. Nat Rev Neurosci 5:97-107.

Turrigiano GG, Lemasson G, Marder E (1995) Selective regulation of current densities underlies spontaneous changes in the activity of cultured neurons. J Neurosci 15:3640-3652.

Turrigiano GG, Leslie KR, Desai NS, Rutherford LC, Nelson SB (1998) Activity-dependent scaling of quantal amplitude in neocortical neurons. Nature 391:892-896.

Uvarov B (1966) Grasshoppers and locusts. A handbook of general acridology. Volume I. Anatomy, physiology, development, phase polymorphism, introduction to taxonomy. Cambridge: Cambridge UP.

Uvarov B (1977) Grasshoppers and locusts. A handbook of general acridology. Volume II. Behaviour, ecology, biogeography, population dynamics. London: Centre for Overseas Pest Research.

Vahasoyrinki M, Niven JE, Hardie RC, Weckstrom M, Juusola M (2006) Robustness of neural coding in Drosophila photoreceptors in the absence of slow delayed rectifier $\mathrm{K}^{+}$channels. J Neurosci 26:2652-2660.

Wilson M (1975) Angular sensitivity of light and dark adapted locust retinula cells. J Comp Physiol 97:323-328. 